\title{
Partial Pixels: A Three-Dimensional Diffractive Display Architecture
}

\section{Gregory P. Nordin}

nordin@byu.edu

M. W. Jones

S. T. Kowel

J. H. Kulick

R. G. Lindquist

See next page for additional authors

Follow this and additional works at: https://scholarsarchive.byu.edu/facpub

Part of the Electrical and Computer Engineering Commons

\section{Original Publication Citation}

J. H. Kulick, G. P. Nordin, A. Parker, S. T. Kowel, R. G. Lindquist, M. Jones, and P. Nasiatka,"Partial Pixels: A Three-Dimensional Diffractive Display Architecture"'" J. Opt. Soc. Am. A, 12(1), pp.

73-83 (1995)

\section{BYU ScholarsArchive Citation}

Nordin, Gregory P.; Jones, M. W.; Kowel, S. T.; Kulick, J. H.; Lindquist, R. G.; Nasiatka, P. J.; and Parker, A., "Partial Pixels: A Three-Dimensional Diffractive Display Architecture" (1995). Faculty Publications. 1156. https://scholarsarchive.byu.edu/facpub/1156 accepted for inclusion in Faculty Publications by an authorized administrator of BYU ScholarsArchive. For more information, please contact ellen_amatangelo@byu.edu. 


\section{Authors}

Gregory P. Nordin, M. W. Jones, S. T. Kowel, J. H. Kulick, R. G. Lindquist, P. J. Nasiatka, and A. Parker 


\title{
Partial pixels: a three-dimensional diffractive display architecture
}

\author{
J. H. Kulick, G. P. Nordin, A. Parker, S. T. Kowel, R. G. Lindquist, M. Jones, and P. Nasiatka \\ Department of Electrical and Computer Engineering, The University of Alabama in Huntsville, Huntsville, Alabama 35899
}

Received January 19, 1994; revised manuscript received July 15, 1994; accepted August 8, 1994

\begin{abstract}
We describe in detail the partial pixel architecture that permits the realization of three-dimensional (3-D) displays that are functionally equivalent to a real-time holographic stereogram. This architecture permits the simultaneous presentation of multiple stereoscopic images so that motion parallax is discernible in the resultant 3-D scene. The key innovation of the architecture is that each pixel is subdivided into partial pixels, which in turn can be implemented as individual diffraction gratings. We describe a static display that exhibits a 3-D image with one-dimensional motion parallax, thereby demonstrating key features of the architecture. A variety of partial pixel implementations are discussed that can operate at video frame rates. These include voltage-controlled liquid crystal gratings and binary optic gratings integrated with conventional liquid crystal amplitude modulators. In addition, we describe how the partial pixel architecture can be generalized for the implementation of full-color displays and displays having two-dimensional motion parallax.
\end{abstract}

\section{INTRODUCTION}

There has been significant interest in recent years in real-time holographic display systems. ${ }^{1-5}$ Such display systems should permit truly three-dimensional (3-D) objects or scenes to be viewed by one or more observers with either one-dimensional (1-D) or two-dimensional (2-D) motion parallax. Potential applications of such systems include mechanical design, training and simulation systems, medical imaging, virtual-reality systems, and architectural design.

There are several significant obstacles to the realization of a truly real-time (i.e., video frame rate) holographic display system. The first is the need for a display device with a huge space-bandwidth product. The second is the requirement for significant computational resources to calculate the requisite grating patterns. ${ }^{4,5}$ In this paper we describe a novel architecture (hereinafter referred to as the partial pixel architecture) that is functionally equivalent to a real-time holographic stereogram. It uses space-bandwidth products that are easily achievable with VLSI technology. It also leads to a significant reduction in the computational load required to drive the display while permitting dedicated processors to be embedded within the display itself to handle those computations that are required. Both 1-D and 2-D motion parallax are obtainable with this architecture, as well as full-color viewing. In contrast to alternative approaches, ${ }^{5}$ the partial pixel concept is well suited to the use of incoherent readout illumination. In addition, displays based on the partial pixel architecture are autostereoscopic because special headgear is not required.

In this paper we discuss the basic concepts of the partial pixel architecture and several methods of physically realizing the architecture. We begin in Section 2 with a brief examination of the major types of holographic display that are currently in use. The partial pixel architecture, which is based on a pixelated display, is then discussed in Section 3. An experimental implementation of a static version of the architecture is examined in
Section 4. Methods of physically implementing real-time controllable partial pixels are discussed in Section 5 , and generalizations of the basic partial pixel architecture to achieve full-color images and 2-D motion parallax are reviewed in Section 6 .

\section{THREE-DIMENSIONAL DISPLAY CONCEPTS}

Static display holograms are made with the use of a variety of techniques and display geometries. For our purpose we classify such holograms into two categories: (1) real-object holograms ${ }^{6}$ and (2) holographic stereograms. ${ }^{7}$

As the name implies, real-object holograms are used to record real, physical, $3-\mathrm{D}$ objects. In the recording process a coherent beam is split into a reference beam and a beam that illuminates the object. At a holographic plate the interference pattern between the reference beam and light scattered or reflected by the object is recorded as a space-variant transmittance or refractive-index modulation. During reconstruction one uses the reference beam to read out the resultant hologram. Light diffracted by the hologram reconstructs a virtual image of the object that is essentially the same size and in the same position as the original object. An observer located in an appropriate viewing region with respect to the holographic plate thus perceives a 3-D object with full motion parallax.

This recording and readout scheme is useful for the reconstruction of monochromatic images from static transmission holograms. Image-plane ${ }^{6}$ and rainbow holograms ${ }^{8}$ involve an additional recording step such that white-light viewable (full-color) holograms are constructed. Both monochromatic ${ }^{6}$ and full-color ${ }^{9}$ reflection holograms can also be produced.

Despite this wide variety of recording and reconstruction techniques, real-object holograms are unsuitable for objects (such as mountains and bacteria) that differ significantly in size compared with that of a typical holographic plate or for objects [such as a computeraided design (CAD) and a tomographic-derived image] 


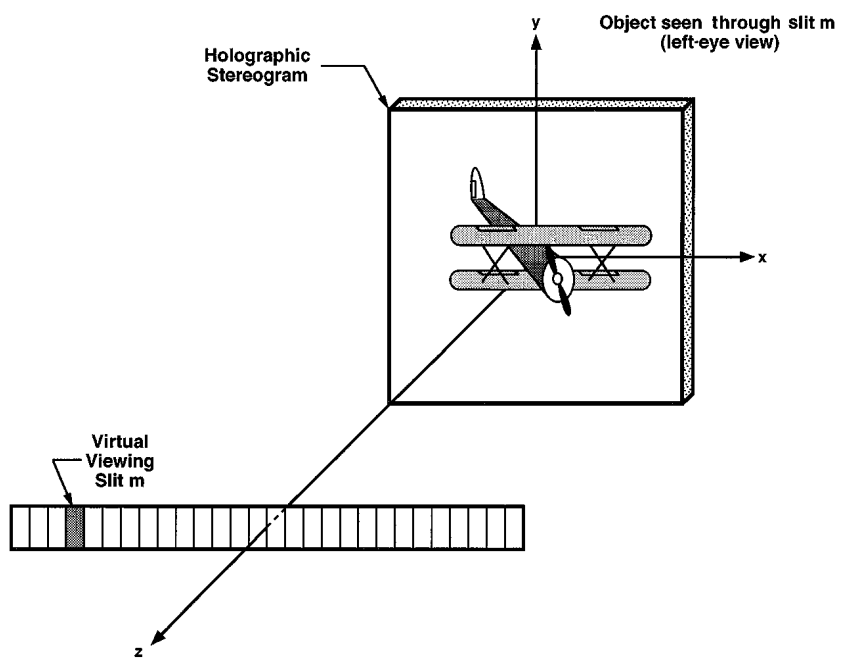

(a)

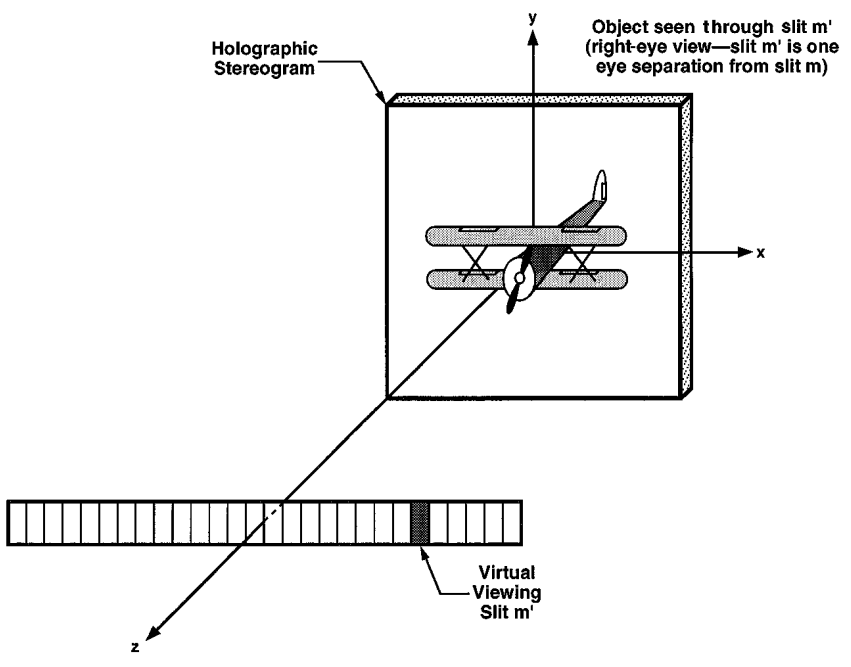

(b)

Fig. 1. Schematic diagram of a holographic stereogram that is viewed from a region that consists of virtual viewing slits. A single 2-D image is visible in the plane of the display when an observer's eye looks through any particular virtual viewing slit. In general, a different 2 -D image can be seen through each virtual viewing slit. When each eye sees the appropriate image of a given pair of stereoscopic images, the observer perceives a 3-D scene. The 2-D images shown in (a) and (b) are intended to illustrate such a pair of stereoscopic images. If an appropriate set of stereopair images is displayed through the virtual viewing slits, 1-D motion parallax is perceived as an observer moves horizontally in the viewing region.

that exist only as a description or a set of data points. For these types of object, holographic stereograms may be constructed. ${ }^{7}$

This form of holographic display involves several recording steps in which a series of stereopair images are recorded. The final hologram reconstructs each recorded image such that it is observable near the plane of the hologram from within only a narrow portion of the viewing region (hereinafter referred to as a virtual viewing slit). As we show in Fig. 1, a given image is visible only through a single virtual viewing slit, which is typically $3 \mathrm{~mm}$ wide (approximately 1 pupil diameter). A different image is visible through each virtual viewing slit. By recording appropriate stereopair images, an observer perceives a 3-D image with 1-D (horizontal) parallax. Thus a holographic stereogram does not reconstruct a true holographic image but, rather, reconstructs a series of 2-D stereopair images. It is this property that permits objects of arbitrary size to be displayed with a holographic stereogram.

Holographic stereograms use at least two of the cues that are normally present in everyday human stereo perception. These are disparity, which is the difference between the left and right eye images as formed on the retinas, and motion parallax. In addition, if the 3-D scene straddles the plane of the holographic plate, several other cues are provided. These include vergence, which is the adjustment of the eye muscles such that both eyes point at the same position in space, and accommodation, in which the eyes are focused in the same general region in which the 3-D scene is perceived. Although the psychophysics of stereo perception are not fully understood, holographic stereograms can be configured to provide a striking sense of visual depth with the use of consistent visual cues. A thorough discussion of such issues can be found in Ref. 10.

An important aspect of nearly all static display holograms (both real-object holograms and holographic stereograms) is that an incoherent illumination source is typically used during reconstruction. The wavelength selectivity and angular diffraction properties of such holograms (which have a thickness of at most several tens of micrometers) diffract the incident light into the appropriate viewing region. Use of an inexpensive incoherent source significantly enhances the convenience of display holograms.

A particularly promising effort to produce a real-time holographic display is described in Refs. 4 and 5. It uses an acousto-optic modulator and thus requires a coherent illumination source. The system employs a unique hololine method of realizing the visual equivalent of a monochromatic real-object hologram. ${ }^{4,5,11,12}$ In the following sections we describe an alternative approach (the partial pixel architecture) that is analogous to a holographic stereogram rather than a real-object hologram. It permits the use of an incoherent illumination source and a flat-panel display format.

\section{PARTIAL PIXEL ARCHITECTURE}

In this section we discuss the basic features of the partial pixel architecture. For simplicity we consider a monochromatic display that exhibits 1-D parallax. (Generalization to full-color displays having 2-D parallax is discussed in Subsection 6.A). We assume that the display is illuminated by a collimated monochromatic beam incident at a fixed orientation relative to the display. In the following subsections, first we discuss pixelation of the display and the functional requirements for individual pixels, then we examine conceptual approaches to fulfilling these requirements, and finally we analyze a pixel implementation that uses area-multiplexed diffraction gratings.

\section{A. Pixel Requirements}

Similar to a holographic stereogram, a display based on 


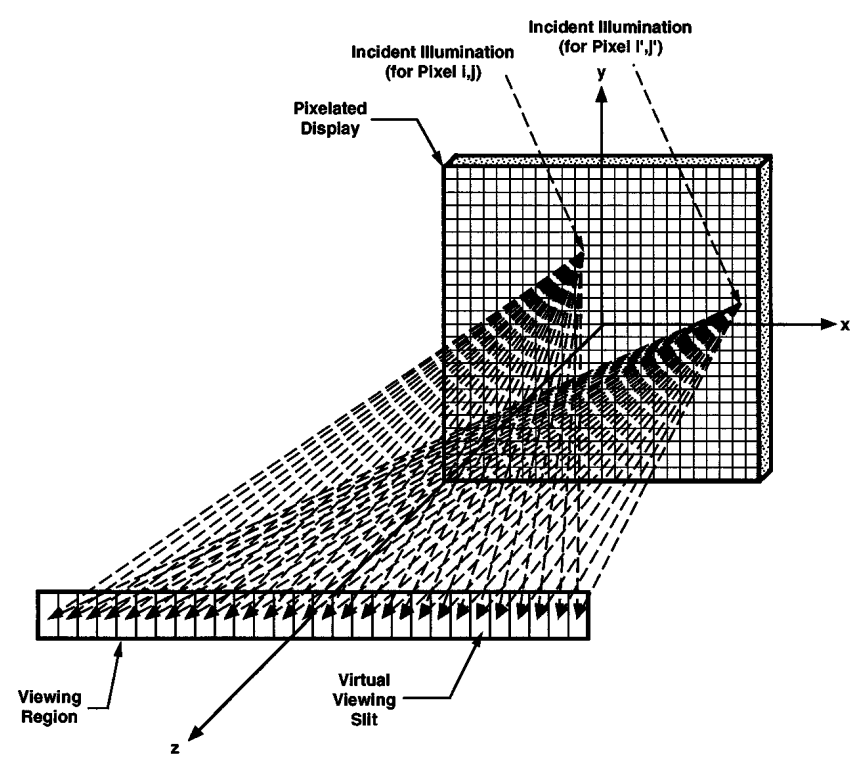

Fig. 2. Schematic illustration showing two display pixels illuminating each of the virtual viewing slits. In general, all the display pixels are able to illuminate each virtual viewing slit simultaneously.

the partial pixel architecture is observed from a viewing region composed of distinct virtual viewing slits, with a different image being visible on the display when it is viewed from each virtual viewing slit. By displaying an appropriate series of stereoscopic image pairs, one can observe 3-D scenes with 1-D parallax.

However, in contrast to most holographic stereograms, the partial pixel display is composed of discrete pixels. Each pixel must therefore have the capability of exhibiting a different appearance when it is viewed from distinct virtual viewing slits (for example, a given pixel might need to appear dark from virtual viewing slit $m$ while appearing bright from virtual viewing slit $m+1$ ). This translates into the following two requirements: (1) each pixel must be capable of directing light into all virtual viewing slits, and (2) each pixel must permit independent control of the intensity of the light that is directed to any given virtual viewing slit. In Fig. 2 we schematically illustrate two pixels (indexed by $i, j$ and $i^{\prime}, j^{\prime}$ ) directing portions of a readout beam into every virtual viewing slit.

\section{B. Conceptual Approaches}

1. Composite Pixel Approach

One approach to fulfilling pixel requirements (1) and

(2) is to multiplex separate holograms within each pixel as schematically illustrated in Fig. 3 (which we refer to as the composite-pixel approach). Each hologram has a fixed grating pattern designed to diffract light from the incident beam to a specific virtual viewing slit. Control of a particular hologram's amplitude would regulate the amount of light that it diffracted to its virtual viewing slit.

Although conceptually straightforward, the compositepixel approach is difficult to realize in practice for at least two reasons. First, a significant space-bandwidth product is required in each pixel to permit multiplexing of numerous holograms, and second, a large dynamic range for the induced multiplexed hologram is needed to permit accurate control over the summed individual hologram amplitudes. We have therefore chosen to develop an alternative approach that is described below.

\section{Partial Pixel Approach}

In the partial pixel architecture each pixel is subdivided into spatially distinct partial pixels, each of which directs light into a single virtual viewing slit. This is schematically illustrated in Fig. 4 for a pixel composed of nine partial pixels that are arranged in a $3 \times 3$ array. Fulfillment of pixel requirements (1) and (2) discussed above can be achieved by the requirement that each partial pixel not only direct light into a single virtual viewing slit but also control the amount of light that is sent to its virtual viewing slit. When this area-multiplexing approach is used, the partial pixel architecture dramatically simplifies the physical realization of a given pixel by reducing the problem to the implementation of spatially distinct partial pixels.

It is important to note that the size of each pixel is designed to be barely resolvable by a human eye located in the viewing region. Consequently, individual partial pixels are not resolvable. Thus, even though the light that is directed to a given virtual viewing slit from a particular pixel comes from only a small region of the pixel (i.e., the appropriate partial pixel of that pixel), it is visually no different than if the light came from the whole pixel.

For the case of a monochromatic display the number of partial pixels is equal to the number of virtual viewing slits. For example, if there are 100 virtual viewing slits, then each pixel must be composed of 100 partial pixels. If each pixel is $1 \mathrm{~mm} \times 1 \mathrm{~mm}$ in size, one possible configuration is to arrange the partial pixels as a $10 \times 10$ array within each pixel, in which case each partial pixel would be $100 \mu \mathrm{m} \times 100 \mu \mathrm{m}$.

The partial pixel architecture has a number of compelling advantages. First, the computational problem is

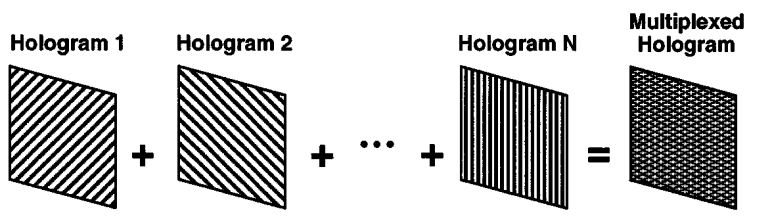

Fig. 3. In the composite-pixel approach multiple holograms are superimposed within the area of any given pixel.

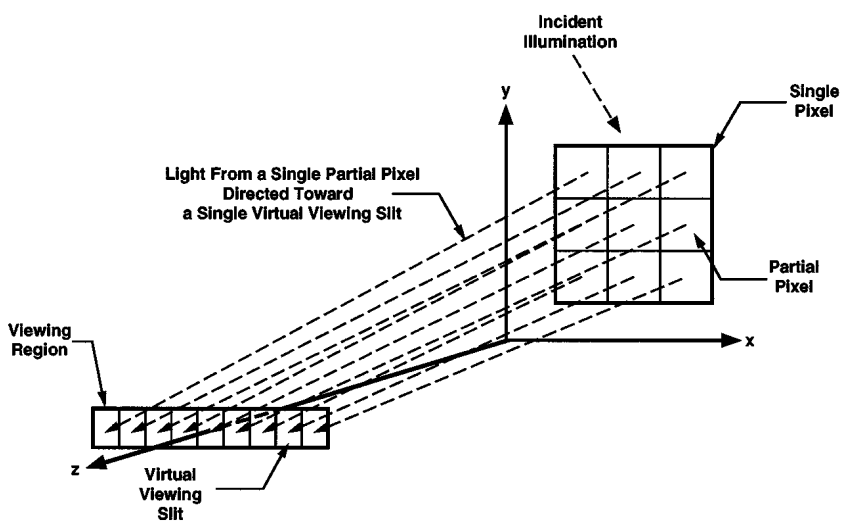

Fig. 4. Schematic representation of a single pixel that is composed of multiple partial pixels, each of which directs light to a single virtual viewing slit. 


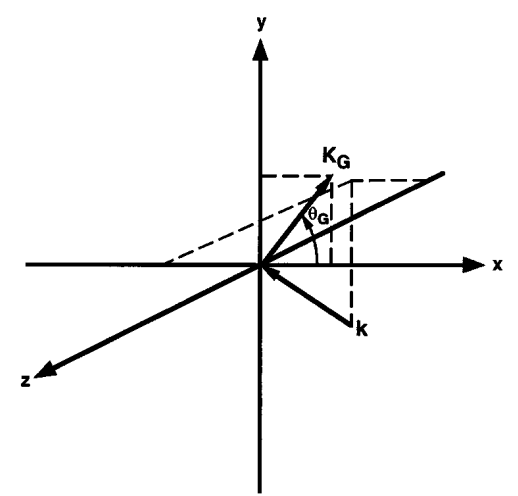

Fig. 5. Arbitrarily oriented collimated readout beam (represented by its $k$ vector) incident upon a grating (also represented by its $k$ vector) that is in the $x-y$ plane.

reduced to simply calculating an appropriate set of 2-D stereographic images instead of trying to calculate holographic fringe patterns. Second, the display problem is physically segregated such that any given partial pixel must direct light in only a single direction. Third, many partial pixel implementations permit the use of incoherent readout illumination. Finally, the spatial segregation of the partial pixels permits significant flexibility in achieving 2-D parallax and full-color images (as discussed in Section 6 below).

One potential disadvantage of the partial pixel architecture is the requirement for large numbers of partial pixels. For example, a $640 \times 480$-pixel display $\left(3 \times 10^{5}\right.$ total pixels) with 40 virtual viewing slits requires $1.2 \times 10^{7}$ partial pixels. Subsection 5.A below describes a potential technique for achieving such large numbers of partial pixels in a real-time display.

\section{Diffraction-Grating-Based Partial Pixels}

One method of implementing the partial pixel architecture is to use a diffraction grating in each partial pixel to diffract light toward its corresponding virtual viewing slit. In this subsection we derive the grating parameters for each partial pixel that we require to achieve this objective (i.e., the grating's period and its angular orientation). In addition, we examine how diffraction from the aperture of a partial pixel can be used to fill its corresponding virtual viewing slit with light from that partial pixel. Methods of modulating the diffracted light are discussed in Section 5 below.

\section{Calculation of Grating Parameters}

Consider the geometry shown in Fig. 5, in which a unitamplitude monochromatic plane wave is incident upon a transmission grating that is constrained to be in the $x-y$ plane (i.e., the plane of the display). We initially assume a sinusoidal phase transmission grating. The incident beam has a wave vector

$$
\mathbf{k}=k(\alpha \hat{x}+\beta \hat{y}+\gamma \hat{z})
$$

in which $k \equiv|\mathbf{k}|=2 \pi / \lambda, \lambda$ is the wavelength of the readout illumination, and $\alpha, \beta$, and $\gamma$ are the vector's direction cosines. The grating is described by its $k$ vector $\mathbf{K}_{G}$ as

$$
\mathbf{K}_{G}=K_{G}\left[\left(\cos \theta_{G}\right) \hat{x}+\left(\sin \theta_{G}\right) \hat{y}\right],
$$

where $K_{G} \equiv\left|\mathbf{K}_{G}\right|=2 \pi / \Lambda, \Lambda$ is the period of the grating, and $\theta_{G}$ is the angle between the grating wave vector and the $x$ axis.

Scalar diffraction theory ${ }^{13}$ can be used in the calculation of the diffracted electric field $U(\mathbf{r})$ in the region $z>0(\mathbf{r}=x \hat{x}+y \hat{y}+z \hat{z})$. The result is

$$
U(\mathbf{r})=\sum_{q=-\infty}^{\infty} J_{q}(v) \exp \left(i \mathbf{k}_{q} \cdot \mathbf{r}\right)
$$

in which $J_{q}$ is the $q$ th Bessel function of the first kind, $v$ is the grating strength (given by $v=2 \pi \Delta n d / \lambda$, in which $\Delta n$ is the amplitude of the grating's refractive-index modulation and $d$ is the grating thickness), and

$$
\mathbf{k}_{q}=k\left(\alpha_{q} \hat{x}+\beta_{q} \hat{y}+\gamma_{q} \hat{z}\right),
$$

with direction cosines

$$
\begin{aligned}
& \alpha_{q}=\alpha+q \frac{\lambda}{\Lambda} \cos \theta_{G}, \\
& \beta_{q}=\beta+q \frac{\lambda}{\Lambda} \sin \theta_{G}, \\
& \gamma_{q}=\left(1-\alpha_{q}{ }^{2}-\beta_{q}{ }^{2}\right)^{1 / 2} .
\end{aligned}
$$

Each term of the sum in Eq. (3) represents a distinct diffraction order that has an amplitude $J_{q}(v)$ and a wave vector $\mathbf{k}_{q}$. Diffraction orders with real $\gamma_{q}$ represent propagating plane waves, and orders having imaginary $\gamma_{q}$ are evanescent. Using Eqs. (5), we can find the propagation direction of the $q$ th diffracted order that is generated by an arbitrarily oriented grating in the $x-y$ plane (as described by its period $\Lambda$ and its angular orientation $\theta_{G}$ ) for any arbitrarily oriented incident beam \{as described by the wavelength of the illumination, $\lambda$, and the collimated beam's direction cosines $\alpha$ and $\beta$ [recall that $\left.\left.\gamma=\left(1-\alpha^{2}-\beta^{2}\right)^{1 / 2}\right]\right\}$. Although this result has been derived for a sinusoidal phase grating, the direction of the diffraction orders as specified in Eqs. (5) is independent of the grating profile.

Given a particular display geometry, we can use Eqs. (5) to derive the period and the orientation of the grating required in each partial pixel of the display. This is accomplished through specification of the direction cosines of both the readout beam and the +1 diffraction order that must be diffracted from each partial pixel to its corresponding virtual viewing slit. In the following discussion $i$ and $j$ specify the $x$ and $y$ indices of the $i j$ th pixel, and the index $m$ refers to the $m$ th partial pixel within a given pixel. Each $m$ th partial pixel is responsible for directing light to the center of the $m$ th virtual viewing slit.

As shown in Fig. 6(a), the center of a given partial pixel is described by the vector $\mathbf{s}_{i j m}$, which can be written as

$$
\mathbf{s}_{i j m}=\mathbf{p}_{i j}+\mathbf{q}_{m}
$$

in which $\mathbf{p}_{i j}$ is the center of the $i j$ th pixel and $\mathbf{q}_{m}$ is a vector describing the center of the $m$ th partial pixel of pixel $i, j$ relative to the pixel center. The vector from 


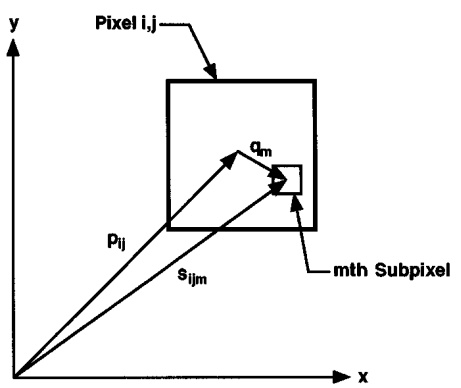

(a)

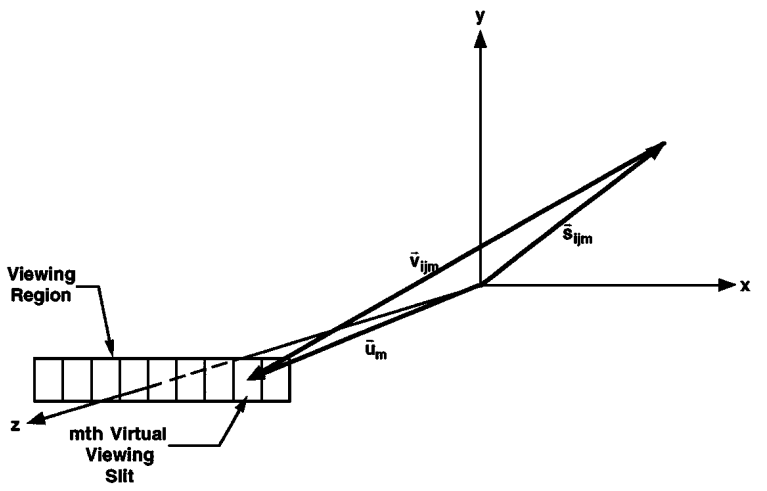

(b)

Fig. 6. (a) Labeling of the vectors that represent the center of each pixel and each partial pixel. (b) Vectors specifying the direction $\left(\mathbf{v}_{i j m}\right)$ in which the +1-order diffracted beam must travel to propagate from partial pixel ijm to the $m$ th virtual viewing slit.

the center of the ijmth partial pixel to the center of the corresponding $m$ th virtual viewing slit, $\mathbf{v}_{i j m}$, is given by

$$
\mathbf{v}_{i j m}=\mathbf{u}_{m}-\mathbf{s}_{i j m},
$$

in which $\mathbf{u}_{m}$ is a vector describing the center of the $m$ th virtual viewing slit [as illustrated in Fig. 6(b)].

The +1 diffraction order from partial pixel ijm must be parallel to vector $\mathbf{v}_{i j m}$, which can be written in terms of its direction cosines as

$$
\mathbf{v}_{i j m}=\left|\mathbf{v}_{i j m}\right|\left(\alpha_{i j m} \hat{x}+\beta_{i j m} \hat{y}+\gamma_{i j m} \hat{z}\right) .
$$

Thus, for a given readout beam (characterized by $\alpha, \beta$, and $\lambda$ ), Eqs. (5) can be solved for the period and the orientation of the grating in the ijmth partial pixel as

$$
\begin{aligned}
\Lambda_{i j m} & =\frac{\lambda}{\left[\left(\alpha_{i j m}-\alpha\right)^{2}+\left(\beta_{i j m}-\beta\right)^{2}\right]^{1 / 2}}, \\
\theta_{G i j m} & =\tan ^{-1}\left(\frac{\beta_{i j m}-\beta}{\alpha_{i j m}-\alpha}\right) .
\end{aligned}
$$

For any display geometry specified by (1) the location of the partial pixels, $\left\{\mathbf{s}_{i j m}\right\}$, (2) the location of the virtual viewing slits, $\left\{\mathbf{u}_{m}\right\}$, and (3) the readout beam wave vector $\mathbf{k}$, Eqs. (9) and (10) can be used to calculate the grating parameters for each partial pixel.

\section{Partial Pixel Aperture Effects}

The above analysis is sufficient for the design of gratings that diffract light from each partial pixel to its corre- sponding virtual viewing slit. Our next concern is that light from any given partial pixel should adequately fill its corresponding virtual viewing slit while not overlapping horizontally into adjacent slits. For the purposes of this subsection we do not consider the visual desirability of some small amount of overlap. Instead, our objective is to discuss a first-order mechanism for controlling the width of the beam diffracted from a given partial pixel as measured at its corresponding virtual viewing slit.

If we plot the intensity of light from a given partial pixel at its virtual viewing slit as a function of the slit's transverse $(x)$ dimension, the ideal beam profile would uniformly fill the slit as shown in Fig. 7. However, diffraction from the aperture of the partial pixel will lead to a nonuniform beam profile. For example, if the partial pixel aperture is rectangular with width $l_{x}$ and height $l_{y}$ such that it can be described by the transmission function $^{13}$

$$
T_{\text {aperture }}(x, y)=\operatorname{rect}\left(x / l_{x}\right) \operatorname{rect}\left(y / l_{y}\right)
$$

in which

$$
\operatorname{rect}(\xi)= \begin{cases}1 & \text { if }|\xi| \leq 1 / 2 \\ 0 & \text { otherwise }\end{cases}
$$

then the intensity in the virtual viewing slit is given by a product of sinc-squared functions:

$$
I_{\text {slit }}(x, y)=I_{0} \operatorname{sinc}^{2}\left(k l_{x} \alpha_{i j m} x / 2 v_{i j m}\right) \operatorname{sinc}^{2}\left(k l_{y} \beta_{i j m} y / 2 v_{i j m}\right),
$$

in which $I_{0}$ is the intensity at the center of the slit, $v_{i j m} \equiv\left|\mathbf{v}_{i j m}\right|$ is the distance from the center of the partial pixel to the center of the slit, the direction cosines $\alpha_{i j m}$ and $\beta_{i j m}$ are $x$ and $y$ obliquity factors, respectively, and

$$
\operatorname{sinc} x \equiv(\sin x) / x \text {. }
$$

The beam profile of Eq. (13) is illustrated in Fig. 7 for $y=0$. [Note that Eq. (13) is valid only when the virtual viewing slit is far enough away for the assumptions of Fraunhofer diffraction to be valid. These approximations are fully satisfied for distances of the order of

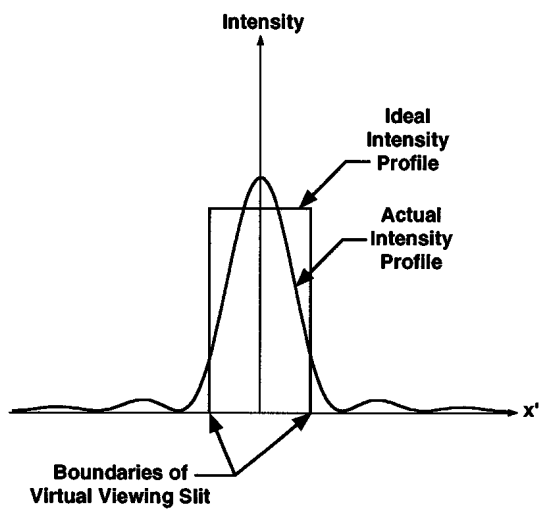

Fig. 7. Ideal and actual (i.e., diffraction-induced) intensity profiles at a single virtual viewing slit that are due to a single partial pixel. 
$100 \mathrm{~mm}$ or greater between the partial pixel and the virtual viewing slit, partial pixel apertures of the order of $100 \mu \mathrm{m}$ wide or less, and visible readout illumination.]

The width of a beam diffracted from a partial pixel to a virtual viewing slit can easily be estimated with Eq. (13). The distance between the first nulls on either side of the peak intensity, $\Delta x$, is approximately (assuming unity obliquity factors)

$$
\Delta x=2 \lambda v_{i j m} / l_{x} .
$$

The full width at half-maximum (FWHM) of the beam can be estimated as $\Delta x / 2$. For example, the FWHM is $3 \mathrm{~mm}$ for a partial pixel aperture of $100 \mu \mathrm{m}$, a readout wavelength of $0.5 \mu \mathrm{m}$, and a viewing distance of $600 \mathrm{~mm}$.

To first order, careful design of the partial pixel apertures can be used to fill each virtual viewing slit with illumination from appropriate partial pixels. This permits the avoidance of the so-called picket fence effect that occurs when the virtual viewing slits are underfilled. However, the presence of sidelobes in the partial pixel aperture diffraction pattern causes some leakage of light into neighboring virtual viewing slits. Whether this significantly affects the visual appeal of the display is a subject for further study. If the sidelobes are found to be undesirable, sidelobe reduction techniques can be examined. Likewise, it is not clear whether the nonuniformity of the main lobe of the diffraction pattern will negatively affect the visual properties of the device. This too will be a subject of future research.

A further point that should be mentioned is that one can similarly use the height $l_{y}$ of the partial pixel apertures to control the height of the virtual viewing slits. Alternatively, other techniques can be used, such as the placement of a cylindrical lens array over the face of the device (for devices with 1-D parallax only)

\section{EXPERIMENTAL DEMONSTRATION}

As described in a previous paper, ${ }^{14}$ we have implemented a static partial pixel architecture based display as a set of amplitude gratings in a chrome mask in order to verify the basic visual properties of the partial pixel approach. We originally reported the observation of a distinct 3-D scene with a flashlight used as an illumination source. In this section we describe results for experiments in which a well-collimated incoherent illumination source was used. This permitted the clear observation of 1-D motion parallax.

The mask device was designed to display the 3-D scene illustrated in Fig. 8. The letter $\mathrm{A}$ is in the plane of the mask, and the letters $\mathrm{U}$ and $\mathrm{H}$ are $6 \mathrm{~mm}$ in front of and behind the mask, respectively. The display region consisted of a $35 \times 35$ array of square pixels covering an area of approximately $1 \mathrm{~cm}^{2}$. As shown in Fig. 9, each pixel contained a $4 \times 8$ array of partial pixels. The number in each partial pixel is the index of the virtual viewing slit to which that partial pixel diffracts light. For a viewing distance of $200 \mathrm{~mm}$ the partial pixel widths were designed to result in minimal overlap of the main diffraction lobes for adjacent virtual viewing slits (i.e., adjacent diffraction lobes overlapped at $18 \%$ of the peak intensity of each lobe).
A side view of the readout geometry of the display is shown in Fig. 10(a). When an observer's eyes are located in the viewing region, the zero-order beam is incident below the observer's nose and thus well out of the viewing region. Likewise, the higher diffraction orders of the partial pixel gratings all propagate above the viewing region and therefore are not visible. The negative diffraction orders are all below the zero order.

Since the gratings in the display were designed for readout illumination at $633 \mathrm{~nm}$, the incident beam was passed through a filter centered at $633 \mathrm{~nm}$ with a spectral bandwidth of $10 \mathrm{~nm}$. In addition, the readout beam was collimated to within $0.1^{\circ}$.

Although 32 partial pixels were available in each pixel, only the top five rows of partial pixels were actually used. As shown in Fig. 10(b), these partial pixels illuminated ten virtual viewing slits for the left eye and ten virtual viewing slits for the right eye. Ten stereoscopic image pairs were therefore visible for individuals with a nominal eye separation of $66 \mathrm{~mm}$. The partial pixel gratings for a single pixel are shown in Fig. 11. This particular pixel encoded one point in the letter A, which was designed to appear in the plane of the display. Hence there is a

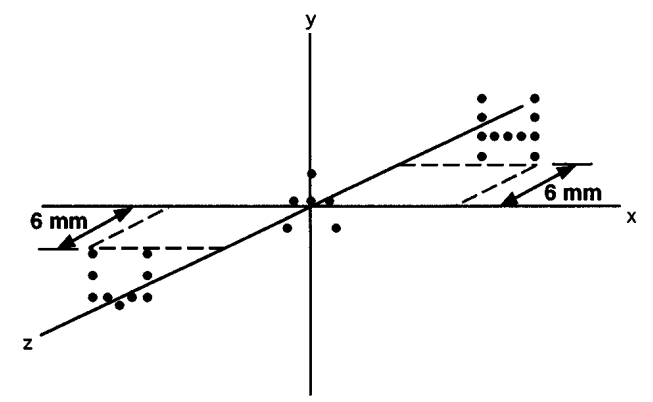

Fig. 8. 3-D object displayed by the chrome mask. Each letter is located in a different plane (all of which are parallel to the $x-y$ plane), and each is composed of a series of dots. The top of the $\mathrm{U}$ and the bottom of the $\mathrm{H}$ are at the $x-z$ plane.

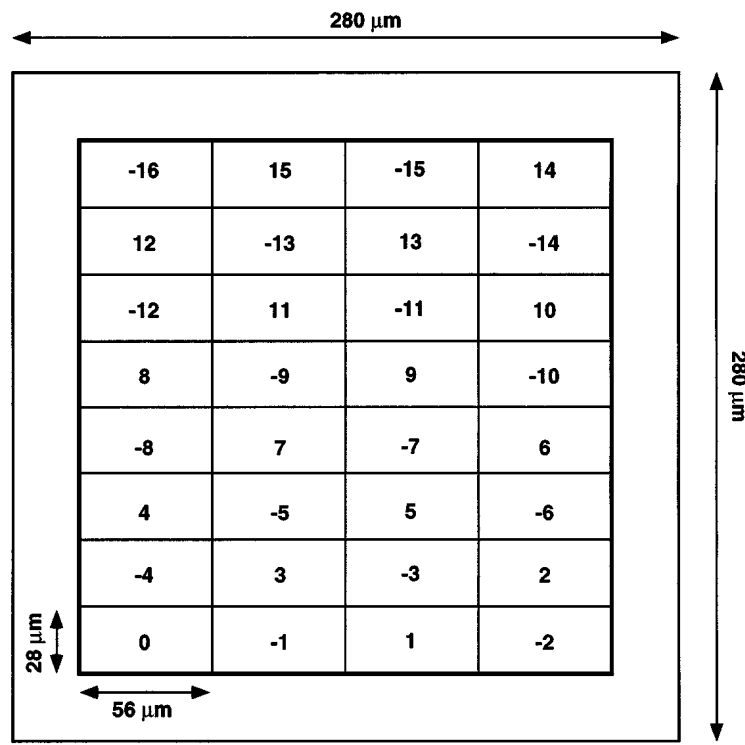

Fig. 9. Schematic illustration of a single pixel that is composed of an array of partial pixels. 


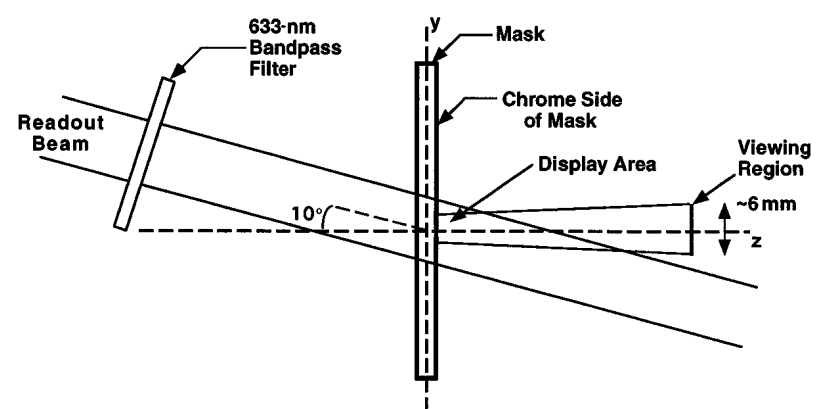

(a)

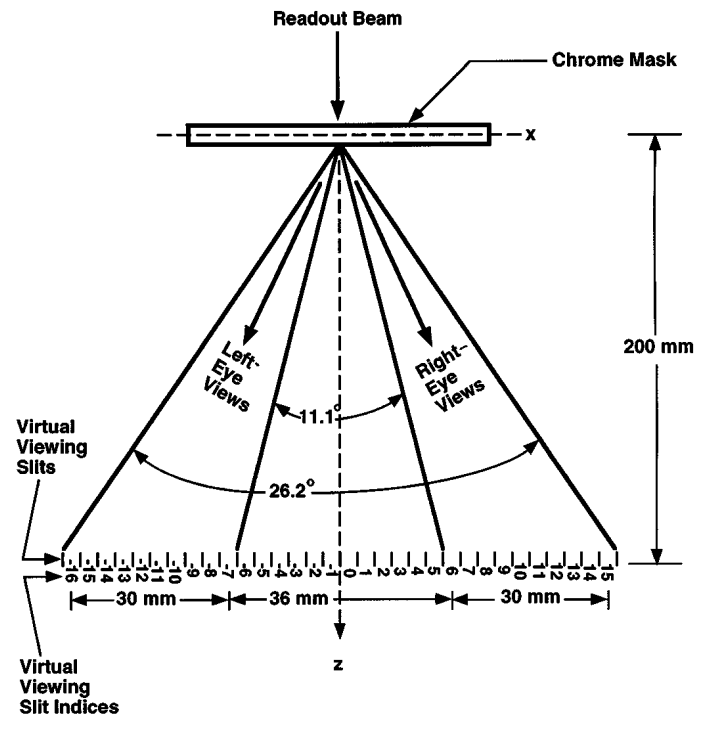

(b)

Fig. 10. (a) Side view of the chrome mask readout geometry. (b) Top view of the readout geometry showing the virtual viewing slits (i.e., slits -16 to -7 and 6 to 15 ) from which images can be observed on the mask.

grating in each of the 20 partial pixels that were used because this pixel must appear bright from each of the 20 virtual viewing slits.

Note that the display was binary in that a given pixel could appear only on or off (corresponding to the presence or the absence of a diffraction grating in a given partial pixel) when it was viewed from a particular virtual viewing slit. For each virtual viewing slit there were 26 partial pixels on the display that contained gratings, since the 3-D scene was composed of 26 points. Thus only 520 partial pixels of a total of 39,200 possible partial pixels had diffraction gratings. The measured diffraction efficiency of the gratings that diffracted light into a given virtual viewing slit was $4.4 \%$. This does not include losses that were due to the nonunity fill factor of each pixel.

When an observer is positioned at the rightmost part of the viewing region (such that the left eye is in virtual viewing slit -7 and the right eye is in virtual viewing slit 15), the observer sees the 2-D images shown in Fig. 12. These readily fuse into the 3-D image of Fig. 8 . As an observer moved horizontally to the left, 1-D motion parallax was apparent as different stereoscopic images were viewed.

Since this device was intended as a simple demonstra- tion of the partial pixel architecture, it was not optimized in terms of optical efficiency, display area, angular viewing range, or degree of observable parallax. For example, the display area on the mask was limited to $1 \mathrm{~cm}^{2}$ so that a single $10 \times$ reticle and exposure step could be used during fabrication of the mask. Larger display areas are easily realizable. Likewise, the angular viewing range can be increased by the use of smaller feature sizes on the mask (the device described above had a minimum feature size of $1 \mu \mathrm{m}$ ). A larger angular viewing range permits greater motion parallax, which could alternatively be obtained by the selection of stereoscopic image pairs that exaggerate the rotation of the 3-D scene. A longer viewing distance is also readily achieved by adjustment of the pixel and partial pixel dimensions.

Fig. 11. Photomicrograph of a single pixel in which there are diffraction gratings in 20 partial pixels (after Ref. 14).

(a)

(b)

Fig. 12. Photographs of stereoscopic images seen through slits (a) -7 and (b) 15 


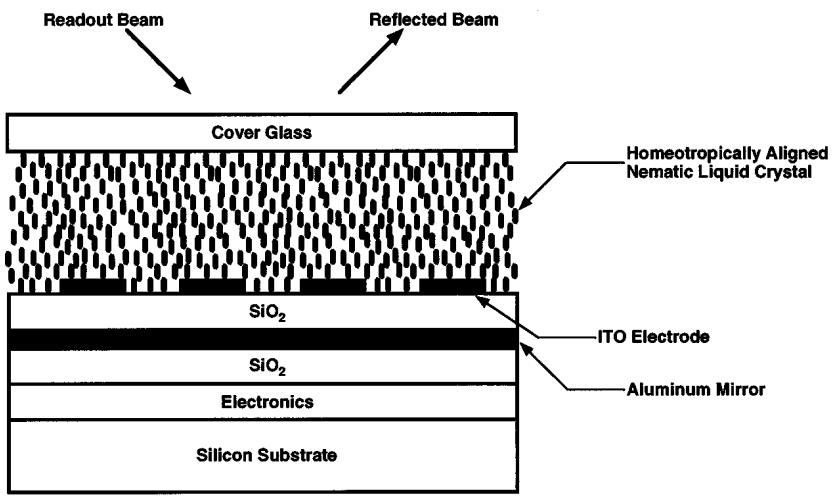

(a)

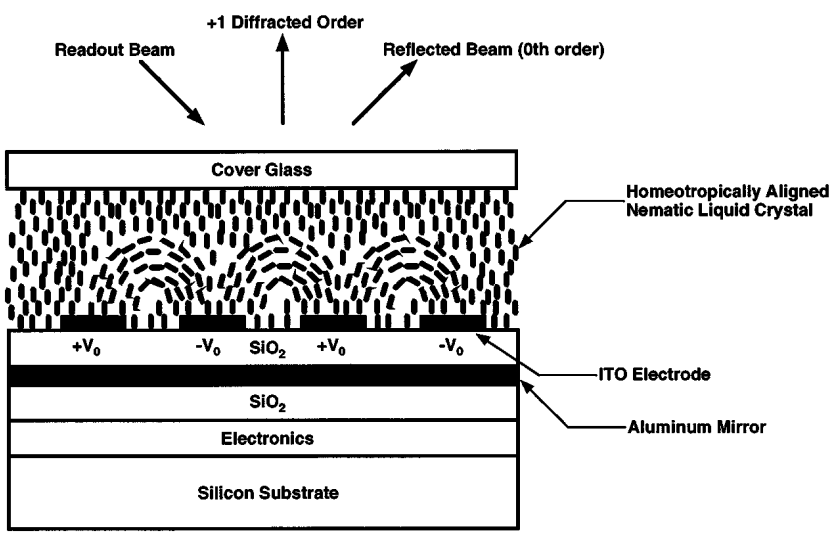

(b)

Fig. 13. Schematic diagram of a liquid crystal film on interdigitated electrodes (a) without an applied voltage and (b) with an applied voltage. The details are discussed in the text. ITO, indium tin oxide.

\section{REAL-TIME PARTIAL PIXEL IMPLEMENTATION CONCEPTS}

In this section we describe various methods of achieving a dynamic display based on the partial pixel architecture. Such a display requires real-time controllable partial pixels. We first discuss the use of variable-amplitude liquid crystal gratings and their incorporation in a display composed of tessellated integrated-circuit dies. We then describe alternative implementations based on binary optic gratings, fixed mirrors or prisms, steerable micromirrors, and deformable gratings. In each of the approaches described below, we use diffraction from the partial pixel apertures to define the spatial extent of the virtual viewing slits in the viewing region.

\section{A. Electronically Controlled Liquid Crystal Gratings}

The chrome mask partial pixels described above fulfill the first partial pixel requirement of directing light into a single virtual viewing slit (discussed in Subsection 3.B.2), but the amount of deflected light is controlled only by the presence or the absence of a fixed diffraction grating. A real-time gray-scale display could be achieved if each partial pixel consisted of a diffraction grating with a controllable amplitude. We recently demonstrated such gratings in thin liquid crystal layers. ${ }^{15}$

The basic idea is illustrated in Fig. 13. A homeotropically aligned nematic liquid crystal film is placed over a set of interdigitated electrodes. When there is no applied voltage between the electrodes, there is no significant diffraction of the readout beam [Fig. 13(a)]. However, application of a voltage results in a fringing electric field between adjacent electrodes, which in turn causes a rotation of the local director axis in the liquid crystal layer [Fig. 13(b)]. The net result is a voltage-controlled diffraction grating having the same period as that of the electrode pitch. A sample pair of interdigitated electrodes for a single partial pixel is shown in Fig. 14.

As illustrated in Fig. 15, a large-area display can be created with tessellated silicon dies ${ }^{16,17}$ each of the order of $1 \mathrm{~cm}^{2}$. An array of pixels would be located on each die, with each pixel consisting of multiple partial pixels. Individual partial pixels would be implemented as shown in Figs. 13 and 14. A major motivation for this approach is that it permits the construction of a large-area display with the use of conventional VLSI fabrication techniques. It also provides the opportunity to locate the display electronics and a graphics computational engine underneath the electrode and liquid crystal layers on each die (as

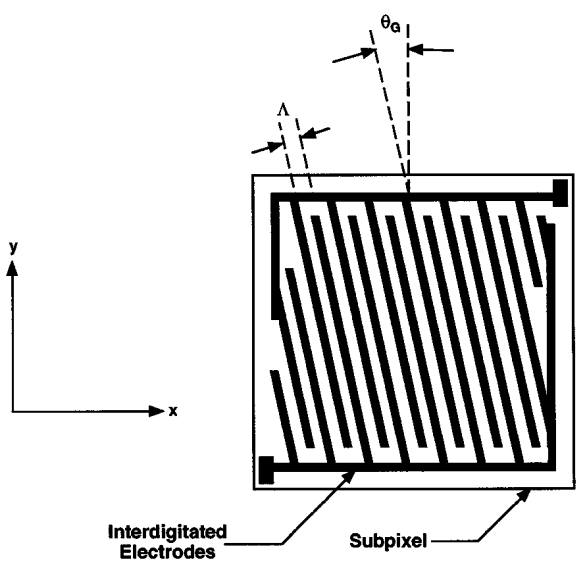

Fig. 14. Schematic diagram of the interdigitated electrodes in a single partial pixel.

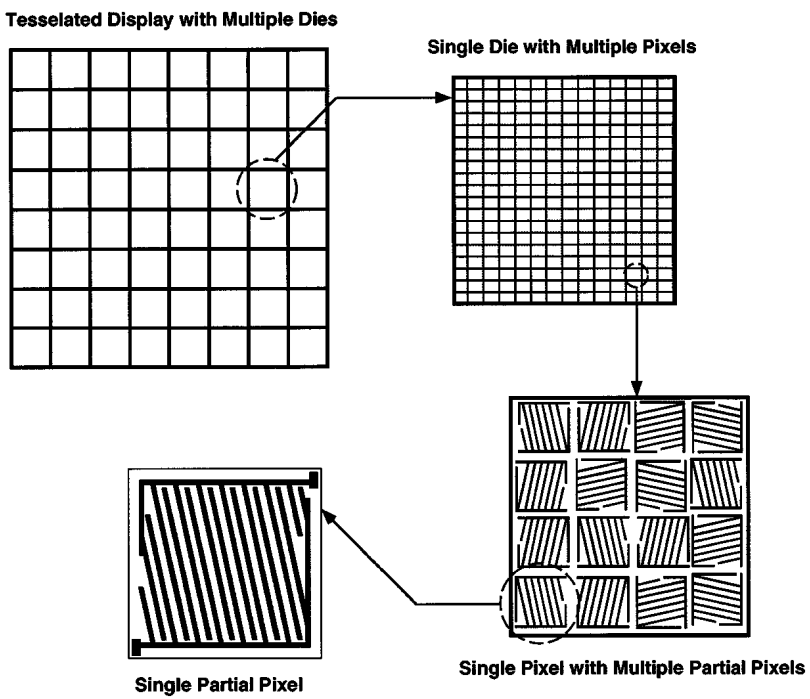

Fig. 15. The diagram in the upper left-hand corner represents a display composed of tessellated silicon dies. The other diagrams are (in clockwise order) a single die on which multiple pixels are implemented, a single pixel composed of an array of partial pixels, and the interdigitated electrodes in a single partial pixel. 


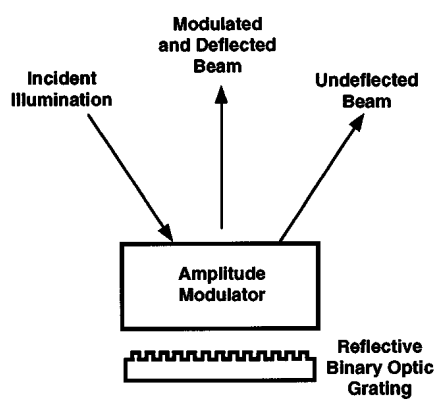

Fig. 16. Schematic representation of a binary optic grating partial pixel.

shown in Fig. 13). Contact to the transparent ITO electrodes would be made with vias through the $\mathrm{SiO}_{2}$ and aluminum mirror layers.

To date, we have realized ITO interdigitated electrodes on silicon with feature sizes as small as $0.4 \mu \mathrm{m}$. We are currently evaluating our first prototype device in which a series of ITO electrodes (with a minimum feature size of $1.0 \mu \mathrm{m}$ ) has been fabricated over an aluminum mirror layer that is on a silicon substrate. This device has been designed to display the same 3-D scene as described above for the chrome mask when a voltage is applied across the electrodes. Preliminary observations indicate that this objective has been achieved. Future efforts will focus on fabricating a full set of interdigitated electrodes over buried drive electronics.

\section{B. Binary Optic Gratings with Modulators}

The liquid crystal grating partial pixel configuration is only one of many possible partial pixel arrangements. For example, the functions of beam deflection and modulation by each partial pixel can be split between two integrated components, rather than both being implemented by a single element such as a liquid crystal grating. Such an alternative design is schematically represented in Fig. 16, in which a reflective binary optic grating is used to deflect the readout beam and a conventional liquid crystal amplitude modulator is used to modulate the beam. The binary optic grating in each partial pixel can be designed with the concepts discussed in Subsection 3.C and would be fabricated in a dielectric layer deposited directly upon the silicon die. A metallic overcoating can be used to make it reflective.

One feature of the binary optic approach is that the modulator element in Fig. 16 is a straightforward application of conventional liquid crystal display technology. In fact, a simple realization of a partial pixel display would be to laminate a conventional liquid crystal display with a binary optic component to form either a transmissive or a reflective device. Each pixel on the liquid crystal display would correspond to a single partial pixel and would be spatially registered with a single grating on the binary optic component.

A further feature of the binary optic approach is flexibility in the design of partial pixel gratings. For example, chirped and nonplanar gratings can be easily realized. In addition, one may use multilevel gratings to achieve increased diffraction efficiency, although at the cost of additional fabrication complexity.
C. Fixed Mirrors or Prisms with Amplitude Modulators An alternative partial pixel configuration is to replace the binary diffraction grating of Fig. 16 with a mirror at a fixed angle and orientation (each of which is different for each partial pixel). The amplitude modulation can be implemented with a liquid crystal modulator as described above. This approach is conceptually equivalent to laminating an array of miniature mirrors to a conventional liquid crystal display such that the mirrors align with the liquid crystal display pixels.

A natural technology for the implementation of the miniature mirror array is diffractive optics, in which optical elements are formed with the use of multilevel phase steps. ${ }^{18}$ Each mirror would be fabricated as a multilevel surface-relief pattern coated with a reflective metallic film. This type of diffractive optic is a reflective kinoform and could be fabricated in a thin-film layer that is deposited directly onto each silicon die.

Alternatively, one could use a prism in series with a flat planar mirror to achieve the same beam deflection function. The prism in each partial pixel would have a different wedge angle and orientation. Such a prism and mirror combination can be fabricated as a diffractive optic kinoform prism over a metallic mirror layer. Both of these layers would reside on top of the silicon die.

\section{Other Approaches}

Another possible partial pixel configuration consists of an electromagnetically deflected micromirror such as those fabricated by Texas Instruments. ${ }^{19}$ Mirrors approximately $16 \mu \mathrm{m}$ square have been constructed with deflections of $\pm 10^{\circ}$. If it is configured as an ICVision ${ }^{16}$ partial pixel, such a mirror could be oriented to deflect light to the appropriate virtual viewing slit when the pixel is supposed to appear in the on position from that slit. When the pixel should appear in the off state, the mirror would be in its opposite position, such that beam deflection to the viewing region does not occur. A major disadvantage of the micromirror approach is that each of the micromirrors would need to have a slightly different deflection angle, which introduces significant fabrication difficulties.

An alternative partial pixel implementation uses deformable gratings, which are described in Ref. 20. These gratings can be in either an on or an off state and exhibit high-speed (30-ns) bistable switching. Such gratings would be designed according to Eqs. (9) and (10). Gray-scale images can be obtained with temporal multiplexing.

\section{GENERALIZATIONS OF THE BASIC PARTIAL PIXEL ARCHITECTURE}

\section{A. Color}

So far our discussion of the partial pixel architecture has been limited to monochrome illumination. The extension to full-color displays is conceptually straightforward. Since both the grating- and kinoform-based partial pixel configurations are dispersive, the basic idea is to use three partial pixels instead of just one partial pixel in every pixel to direct light to a given virtual viewing slit. Each of the three new partial pixels (hereinafter referred to as primary-color partial pixels) is designed to 


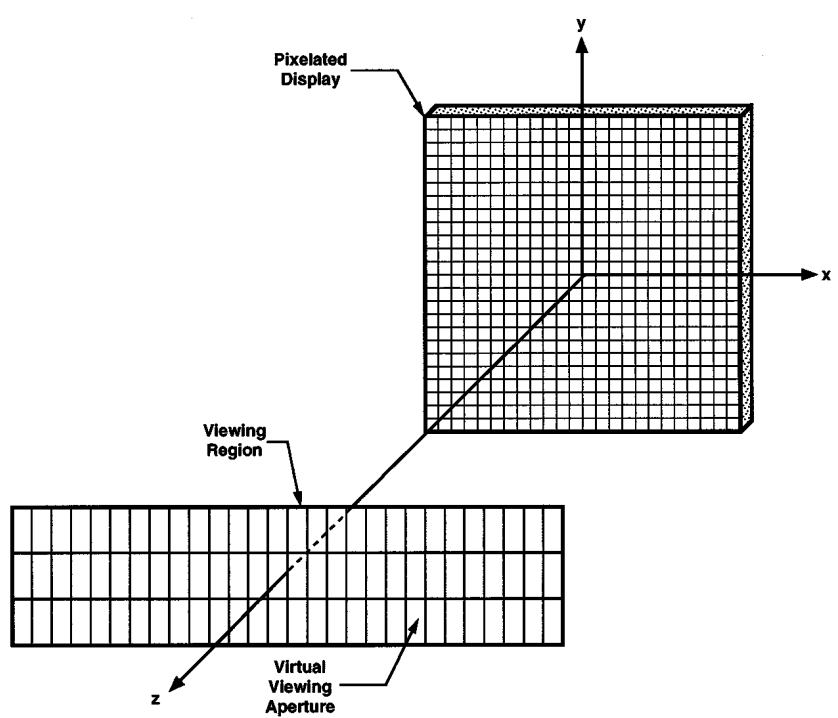

Fig. 17. Display geometry for 2-D motion parallax.

direct a different primary color to the center of the desired viewing slit. This means that each primary-color partial pixel will have, in general, a different grating period for grating-based partial pixels or a different step depth for kinoform-based partial pixels.

If the same number of virtual viewing slits is to be illuminated, there must be three times as many partial pixels as in a monochrome display. Each type of primary-color partial pixel may also have a different aperture size, because the diffraction-induced spreading of the light from any partial pixel is also dispersive.

For overhead white-light illumination the primary-color partial pixel approach should result in the observation of full-color images from the viewing region. This method of achieving a full-color display is analogous to that used in full-color holographic stereogram displays. ${ }^{7}$

\section{B. Two-Dimensional Parallax}

Extension of the partial pixel architecture to a display that has 2-D parallax is also conceptually straightforward. For 2-D parallax the display must show different images for vertical as well as horizontal movement within the viewing region. Instead of a 1-D arrangement of virtual viewing slits, the viewing region must therefore consist of a 2-D set of virtual viewing apertures as shown in Fig. 17. For monochrome displays each pixel in the display must have a partial pixel that directs light to a single virtual viewing aperture. Control of the partial pixel apertures in the vertical as well as the horizontal direction becomes important to prevent light from spreading into vertically adjacent apertures.

Since vertical parallax is visually not as important as horizontal parallax, relatively few rows of virtual viewing apertures are required. For example, a satisfactory display may consist of five rows with each having 50 apertures. This means that 250 partial pixels are required per pixel, which is still a reasonable quantity for many display geometries. The upper bound on the number of realizable partial pixels is set (for a given display geometry and size) by the width of the diffracted beams and the desired size of the virtual viewing apertures. If the partial pixels are too small, the diffracted beams become larger than the virtual viewing apertures.

Generalizing to a full-color display having 2-D parallax is challenging for at least two reasons. First, a factor-of3 more partial pixels are required than for a monochrome display if the number of virtual viewing apertures is kept constant. Second, the dispersion from each partial pixel must be limited for vertical overlapping of dispersed light in different apertures to be avoided. One potential solution is to place an appropriate color filter over each primary-color partial pixel. The search for adequate solutions to both of these issues is a topic for future research.

\section{SUMMARY}

We have described a new method of realizing a 3-D autostereoscopic display that is functionally equivalent to a holographic stereogram and thus naturally provides for 1-D motion parallax. The key to the partial pixel architecture is the concept of pixels composed of an array of spatially distinct partial pixels. Each partial pixel is responsible for how its corresponding pixel appears from a single virtual viewing slit. We have discussed the design of partial pixels based on individual diffraction gratings and have demonstrated a static realization of the partial pixel architecture, using amplitude gratings in a chrome mask. We have also discussed methods of implementing dynamic partial pixels for use in real-time displays. In addition, we have shown how the partial pixel architecture can be extended to the realization of full-color displays and how 2-D motion parallax can be achieved.

We are currently pursuing a range of development activities for several partial pixel implementation concepts, including voltage-controlled liquid crystal gratings on silicon and binary optic gratings integrated with liquid crystal amplitude modulators. Further research directions include packaging of tessellated dies, implementation of buried electronics, planarization of a dielectric layer above the electronic circuits (to provide for a planar mirror layer), methods of communicating graphics data to the buried display processors, and algorithm and software development for driving the display.

\section{ACKNOWLEDGMENTS}

This research was supported by the U.S. Army Missile Command and the Defense Advanced Research Projects Agency. We gratefully acknowledge the assistance of Richard Ciliax and Mark Friends.

\section{REFERENCES}

1. P. St. Hilaire, S. A. Benton, M. Lucente, M. L. Jepson, J. Kollin, H. Yoshikawa, and J. Underkoffler, "Electronic display system for computational holography," in Practical Holography IV, S. A. Benton, ed., Proc. Soc. Photo-Opt. Instrum. Eng. 1212, 174-182 (1990).

2. P. St. Hilaire, S. A. Benton, M. Lucente, H. Yoshikawa, and J. Underkoffler, "Real-time holographic display: improvements using a multichannel acousto-optic modulator and holographic optical elements," in Practical Holography V, S. A. Benton, ed., Proc. Soc. Photo-Opt. Instrum. Eng. 1461, 256-261 (1991).

3. S. A. Benton, "Experiments in holographic video imaging," in Industrial Applications of Holographic and Speckle Mea- 
suring Techniques, W. P. Jueptner, ed., Proc. Soc. Photo-Opt. Instrum. Eng. 1508, 247-267 (1991).

4. M. Lucente, "Optimization of hologram computation for realtime display," in Practical Holography VI, S. A. Benton, ed. Proc. Soc. Photo-Opt. Instrum. Eng. 1667, 32-43 (1992).

5. P. St. Hilaire, S. A. Benton, and M. Lucente, "Synthetic aperture holography: a novel approach to three-dimensional displays," J. Opt. Soc. Am. A 9, 1969-1977 (1992).

6. G. Saxby, Practical Holography (Prentice-Hall, Englewood Cliffs, N.J., 1988).

7. S. A. Benton, "Survey of holographic stereograms," in Processing and Display of Three-Dimensional Data, J. J. Pearson, ed., Proc. Soc. Photo-Opt. Instrum. Eng. 367, $15-19$ (1982)

8. S. A. Benton, "The mathematical optics of white light transmission holograms," in Proceedings of the International Symposium on Display Holography (Holography Workshops, Lake Forest College, Lake Forest, Ill., 1982).

9. J. Walker, "In-situ color control for reflection holograms," Ph.D. dissertation (Massachusetts Institute of Technology, Cambridge, Mass., 1987).

10. D. B. Diner and D. H. Fender, Human Engineering in Stereoscopic Viewing Devices (Plenum, New York, 1993).

11. D. Leseberg and O. Bryngdahl, "Computer-generated rainbow holograms," Appl. Opt. 23, 2441-2447 (1984).

12. D. Leseberg, "Computer-generated holograms: display using one-dimensional transforms," J. Opt. Soc. Am. A 3, 1846-1851 (1986).
13. J. W. Goodman, Introduction to Fourier Optics (McGrawHill, San Francisco, Calif., 1968).

14. G. P. Nordin, J. H. Kulick, M. Jones, P. Nasiatka, R. G. Lindquist, and S. T. Kowel, "Demonstration of a novel 3-D autostereoscopic display," Opt. Lett. 19, 901-903 (1994).

15. R. G. Lindquist, J. H. Kulick, G. P. Nordin, J. M. Jarem, S. T. Kowel, M. Friends, and T. M. Leslie, "High-resolution liquid crystal phase grating formed by fringing fields from interdigitated electrodes," Opt. Lett. 19, 670-672 (1994).

16. J. Kulick, S. T. Kowel, T. Leslie, and R. Ciliax, "ICVision-a VLSI based holographic display system," in Practical Holography VII: Imaging and Materials, S. A. Benton, ed., Proc. Soc. Photo-Opt. Instrum. Eng. 1914,219-229 (1993).

17. J. H. Kulick, S. T. Kowel, G. P. Nordin, A. Parker, R. Lindquist, P. Nasiatka, and M. Jones, "ICVision-a VLSIbased diffractive display for real-time display of holographic stereograms," in Practical Holography VIII, Stephen Bentson, ed., Proc. Soc. Photo-Opt. Instrum. Eng. 2176, 2-11 (1994).

18. O. Bryngdahl and F. Wyrowski, "Digital holographycomputer-generated holograms," in Progress in Optics, E. Wolf, ed. (North-Holland, New York, 1990), Vol. 28 , pp. $3-85$.

19. J. M. Younse, "Mirrors on a chip," IEEE Spectrum 30(11), 27-31 (1993)

20. O. Solgaard, F. S. A. Sandejas, and D. M. Bloom, "Deformable grating optical modulator," Opt. Lett. 17, 688-690 (1992). 


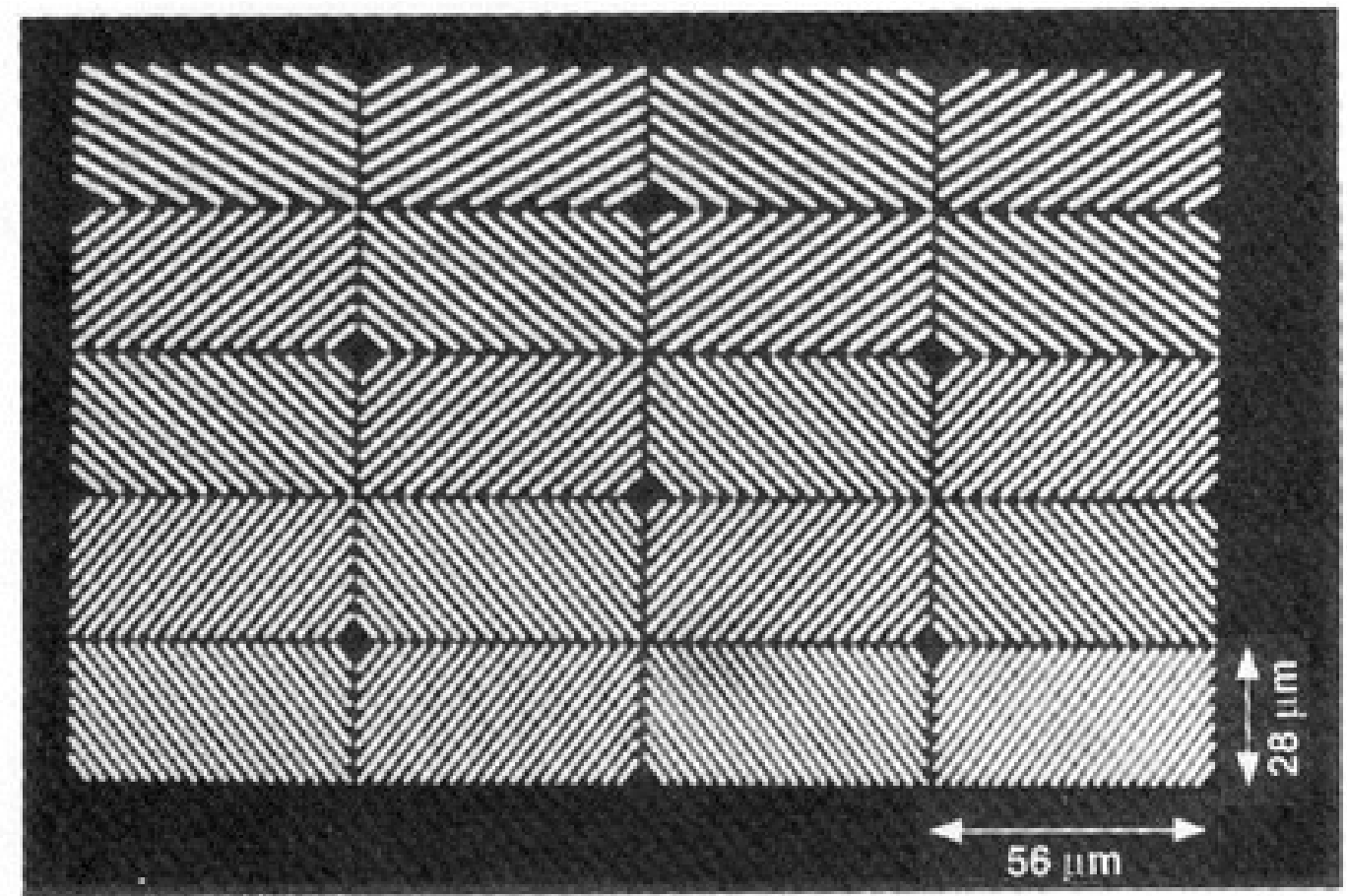


(a)

(b) 